\title{
Pullout modelling of viscoelastic synthetic fibres for cementitious composites
}

\author{
Sorzia A. ${ }^{\mathrm{a}}$, Lanzoni L. ${ }^{\mathrm{b}}$, Radi E. ${ }^{\mathrm{a}, \mathrm{c}}$ \\ ${ }^{a}$ DISMI - Department of Sciences and Methods for Engineering, \\ University of Modena and Reggio Emilia, 42122 Reggio Emilia, Italy \\ ${ }^{b}$ DIEF - Department of Engineering "Enzo Ferrari", \\ University of Modena and Reggio Emilia, 41125 Modena, Italy \\ ${ }^{c}$ En $\&$ Tech
}

\begin{abstract}
The problem of the pullout of a viscoelastic synthetic fibre embedded in a cementitiuos matrix and subjected to an external time-dependent axial load is considered in the present work. A 1D phenomenological model able to simulate the contribution of viscoelastic relaxation as well as the hardening behavior due to abrasion phenomena during slippage is developed. The cement matrix compliance is neglected with respect to the fibre elongation. The interfacial shear stress between the fibre and the surrounding matrix is assumed to depend on the slippage distance through a second degree polynomial law, thus involving three constitutive parameters. Two distinct phases are recognized: An earlier debonding stage followed by the effective fibre pullout process. Two different creep functions have been assumed for modelling the viscous response of polymeric fibres: A function based on the fraction-exponential Rabotnov operator and a classical exponential model. Identification of the governing constitutive parameters allows obtaining the relation between the external strain and the axial displacement, which has been compared with experimental results provided by pullout tests both on plain and treated fibres, finding a good agreement. It is shown that the proposed approach can predict the whole pullout process of discrete synthetic macrofibres.
\end{abstract}

Keywords: Pull-out, Fibre reinforced concrete, Synthetic fibres, Creep, Rabotnov operator, Analytical modelling.

\section{Introduction}

Concrete is widely used in civil engineering because its versatility and cheapness as compared with other building materials like steel and masonry. During the last decades, concrete technology has known a rapid improvement in order to obtain cementitious-based materials with specific physical properties, like

*Corresponding Author: luca.lanzoni@unimore.it 
lightweight concrete, ultra-high-performance concrete (UHPC), self-compacting concrete and foamed concrete (e.g. Scerrato et al. [1]). Despite these advantageous properties, concrete is a brittle material undergoing various damaging phenomena like crack initiation and growth, especially under the action of tensile stresses and impact loads (aging effects in concrete structures have been discussed in [2], [3]). This detrimental aspect can be mitigated by inserting proper reinforcements during the cast of the mixture, like traditional steel bars, brackets ribbed, wire meshes etc., or by introducing discrete fibres in the concrete mixture at the mixing stage [4]. Indeed, fibres provide a uniform reinforcement as they are randomly distributed in the concrete cast, allowing increasing the tensile resistance, ductility and, in turn, its lifespan $[5,6]$. In particular, synthetic polypropylene (PP) macrofibres have proved to impart a significant toughness to the concrete, increasing its durability and mechanical performances in time [7]. Moreover synthetic macrofibres offer many advantages in terms of lightness, cheapness, magnetic permeability and chemical stability in aggressive environments as compared with the metallic ones. Recent studies about the mechanical performances of fabric-reinforced cementitious matrix composites can be found in $[8,9,10,11]$.

The strengthening contribution of macrofibres embedded in a brittle concrete matrix is mainly due to their capacity of transferring stresses across the crack surfaces, thus mitigating the tendency of stress to concentrate under increasing loads by creating a crack bridging mechanism. Such a mechanism is the working principle of FRC $[12,13]$. Under proper conditions, it allows FRC to display hardening post-cracking behavior, thus increasing both the ultimate bearing capacity and toughness of FRC structural elements [5, 14, 15]. These beneficial effects strongly depend on the nature of the fibre-matrix interface, namely the non-homogeneous region of the cement matrix which originates just around the fibre, known as interface transition zone (ITZ) [16].

Recently, Di Maida et al. [17] performed pull-out and flexural experimental tests on FRC based on macro-synthetic fibres, both for plain fibres and for fibres treated with nanosilica on their surface in order to improve the adhesion to the cementitious matrix. They observed slip hardening behavior, namely an increase in the frictional stress acting on the fibre surface as the fibre is pulled out of the cement matrix caused by the progressive wearing of the fibre surfaces and the accumulation of wear debris due to abrasion phenomena. The pullout hardening behavior is responsible, in turn, of the increase in the residual strength during the post-cracking phase and, thus, of the ductile behavior of FRC [18].

A number of analytical and numerical studies have been devoted to simulate the pullout response of various kind of fibres focussing on the mechanism of stress transfer between the fibrous reinforcements and the cement matrix. A simple frictional analytical model for the characterization of the stress-slippage relationship of steel fibres embedded in cementitious matrices was proposed by Naaman et al. [19]. Later, Cunha et al. [20] proposed some bond-slip relationships reproducing the experimental pullout behavior of both straight and hooked steel fibres. A numerical approach has been adopted also by Choi et al. [21], in 
order to characterize the interface between the fibre and the cementitious matrix for three kinds of fibres: carbon fibre, polypropylene (PP) fibre and twisted wire strand steel cord. Radi et al. [22] developed a 1D phenomenological model for simulating the pullout behavior of synthetic fibres. The latter Authors neglected the Poisson effect on the fibre pullout because it would provide softening behavior, whereas the main effect highlighted by the experimental results was hardening frictional behavior. They also neglected the matrix compliance as compared to the fibre elongation, but assumed large deformations of the polymeric fibre and imposed the balance conditions in the deformed configuration. Moreover, they assumed the interfacial shear stress as a second degree piecewise function of the slippage.

It must be remarked that the viscous behavior of the fibre has been neglected in all the aforementioned references. However, the overall mechanical behavior of FRC is expected to be strongly influenced by the rheological properties of synthetic fibres, as pointed out in [5, 23]. Indeed, the viscous relaxation typical of polymeric materials may provide a significant contribution in the relation between the applied tensile load and displacement of the actuated fibre cross section measured by pullout tests. In particular, the pullout response is expected to be strongly affected by viscous effects taking place in the outer part of fibre, namely between the cement sample and the actuator, whereas the viscous deformation of the embedded part of the fibre can be neglected due to the constraint provided by the surrounding rigid matrix. Therefore, in order to validate the analytical model for the pull-out response of polymeric fibres, it becomes necessary to take into considerations also rheological properties of the free part of the fibre.

In the present work, the approach proposed by Radi et al. [22] for the simulation of the hardening behavior exhibited in pullout tests of synthetic fibres has been extended to account for the viscous behavior of the outer part of the fibre, within the framework of hereditary linear viscoelasticity. A general creep function compliant with the Rabotnov viscoelastic operator as well as a simplified creep function following to the idealized Zener viscoelastic scheme, called Standard Linear Solid (SLS), have been considered. The use of fractionexponential operators, like the Rabotnov one, allows describing experimental data of real materials with sufficient accuracy and, at the same time, allows finding explicit analytical results.

The interfacial shear stress between the fibre and the surrounding matrix is assumed to depend on the slippage distance through a second degree polynomial law, thus involving three constitutive parameters. The balance condition for the fibre leads to a nonlinear ordinary differential equation, which is solved through a numerical procedure. The constitutive parameters characterizing the frictional interface behavior as well as the rheological response of the fibre have been determined by comparing the relations between the pull-out load and the displacement of the actuated fibre cross section provided by theoretical simulations with those obtained from the test performed by Di Maida et al. [17]. The theoretical pull-out curves are then found to agree well with the experimental results, both for plain fibres and for fibres treated with nano- 
${ }_{122} x$ axial abscissa

${ }_{123} \varepsilon$ axial strain of the fibre

${ }_{124} \lambda$ parameter describing the debonding phase, $\lambda \in[0,1]$

${ }_{125} \alpha, \beta, \nu$ parameter of the creep function

${ }_{126} \sigma$ tensile stress in the fibre

${ }_{127} \tau$ shear stress at the interface

${ }_{128} \tau_{0}, a, b$ parameters of shear stress law of the interface 
$\psi(t)$ creep function at time $t$

$\psi_{0}$ creep function at time $t=0$

$\psi_{\infty}$ creep function at time $t \rightarrow \infty$

$k_{1}, k_{2}, c$ constant parameters

\section{Governing equations}

\subsection{Constitutive model for the shear stress interface}

In order to simulate the pull-out process of polymeric fibres from the cement matrix a 1D analytical model is developed here by neglecting the deformation of the cement matrix and imposing the equilibrium of the fibre in the undeformed configuration. The fibre is assumed to display linear visco-elastic behavior under small strains. An abscissa $x$ measured along the fibre is taken, with the origin at the embedded end of the fibre and moving with it. A suitable law for the frictional shear stress arising between the fibre and the surrounding matrix is considered. In particular, by denoting with $s(x)$ the slippage distance of the fibre section placed at a generic abscissa $x$, according to Radi et al. [22] the shear stress is assumed as a non-linear function of the slippage, namely

$$
\tau(s)=\tau_{0}+a s+b s^{2}
$$

This constitutive relation for the interface is plotted in Fig. 1. Since the cement matrix is assumed as rigid, then the slippage $s(x)$ coincides with the axial displacement of the fibre cross section at abscissa $x$. The elastic deformation of the interface considered in Radi et al. [22] has been neglected here, because its effect on the whole process of the fibre extraction was found negligible. Thus, according to eqn $(1), \tau(0)=\tau_{0} \neq 0$.

Reference is made to a fibre embedded in a matrix for a length $L$ and subjected to the tensile load $F$. Let $s_{L}$ be the displacement of the fibre cross section initially at $x=L$, namely on the surface of the cement sample, as illustrated in Fig. 2. As the load $F$ increases, two distinct phases occur during the pullout process. At first, a debonding stage originates in the embedded part of the fibre of length $(1-\lambda) L$, with $0 \leq \lambda \leq 1$, starting from the outer side, as reported in Fig. 2(a). As the load increases, the bonded part $\lambda L$ of the fibre decreases till $\lambda=0$. At the end of the debonding phase the effective pullout stage takes place. As a consequence, a rigid body motion $s_{0}$ occurs at $x=0$, starting from $s_{0}=0$ (corresponding to $\lambda=0$ ) till the complete extraction of the fibre, which is reached as soon as $s_{0}=L$ (see Fig. 2(b)).

\subsection{Strain-displacement relation of the fibre}

Let $d$ denotes the diameter of the fibre cross section. Making reference to Fig. 2 and supposing that no axial load acts on the fibre cross section at $x=0$, 


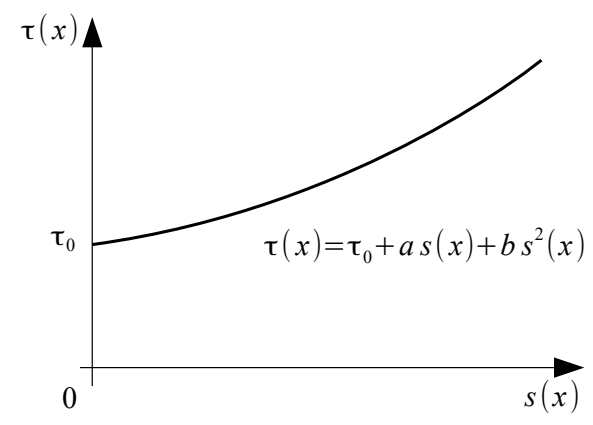

Figure 1: Constitutive model between interface shear stress $\tau(x)$ and slippage distance $s(x)$ at abscissa $x$.

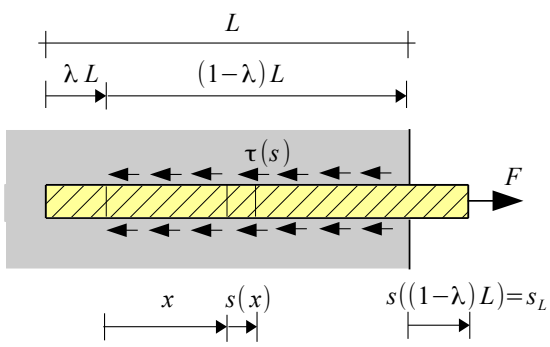

(a)

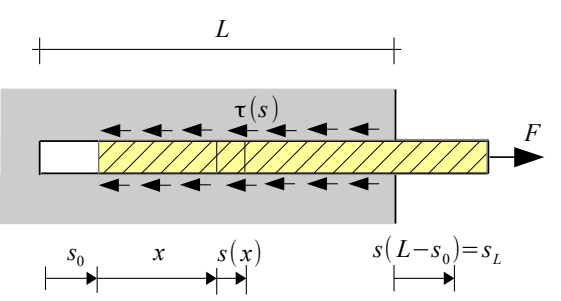

(b)

Figure 2: (a) Debonding phase. (b) Pullout phase. 
the tensile stress $\sigma(x)$ acting on the cross section of the fibre at abscissa $x$ is given by balance condition as

$$
\sigma(x)=\frac{4}{d} \int_{0}^{x}\left[\tau_{0}+a s(\chi)+b s^{2}(\chi)\right] d \chi,
$$

where $0 \leq x \leq L(1-\lambda)$ in debonding stage and $0 \leq x \leq L-s_{0}$ in pullout stage. Let

$$
\varepsilon(x)=\frac{\sigma(x)}{E}=s^{\prime}(x),
$$

denotes the axial strain of the fibre, where the apex denotes derivative with respect to the function argument. By deriving $\varepsilon(x)$ with respect the spatial coordinate $x$, eqns (2) and (3) provide

$$
s^{\prime \prime}(x)-B s^{2}(x)-A s(x)=C,
$$

where the prime denotes differentiation with respect to the argument function and

$$
A=\frac{4 a}{E d}, \quad B=\frac{4 b}{E d}, \quad C=\frac{4 \tau_{0}}{E d} .
$$

By using the definition (3) of the axial strain, eqn (4) becomes a nonlinear ordinary differential equation for the function $\varepsilon$ of the displacement $s$, namely

$$
\varepsilon \frac{d \varepsilon}{d s}-B s^{2}-A s=C .
$$

By integrating eqn (6) with respect to $s$ and then with respect to $x$ one finds the following nonlinear relation between $\varepsilon$ and $s$

$$
\varepsilon(x)=\sqrt{2 C s(x)+\frac{2}{3} B s^{3}(x)+A s^{2}(x)+2 C_{0}},
$$

that can be solved for $x(s)$ after integrating between $x_{0}$ and $x(s)$, namely

$$
\int_{x_{0}}^{x} d x=\int_{s\left(x_{0}\right)}^{s(x)} \frac{d s}{\sqrt{2 C s+\frac{2}{3} B s^{3}+A s^{2}+2 C_{0}}} .
$$

Proper boundary conditions are imposed for each phase. In the debonding phase the boundary conditions at $x=0$ require $s(0)=0$ and $\varepsilon(0)=0$, thus from eqn (7) one obtains $C_{0}=0$, and from eqn (8), for $x_{0}=0$ and $x=L-\lambda L$, one gets

$$
\lambda=1-\frac{1}{L} \int_{0}^{s_{L}} \frac{d s}{\sqrt{2 C s+\frac{2}{3} B s^{3}+A s^{2}}},
$$


where $s_{L}=s(L-\lambda L)$. By varying $\lambda$ in the range $[0,1]$, the corresponding values of the displacement $s_{L}$ are assessed from eqn (9). Then, the corresponding axial strain $\varepsilon_{L}=\varepsilon(L-\lambda L)$ is found from eqn (7).

In the pullout phase the boundary conditions at $x=0$ require $s(0)=s_{0}$ and $\varepsilon(0)=0$, thus from eqn $(7)$ one obtains

$$
C_{0}=-\left(C s_{0}+\frac{B}{3} s_{0}^{3}+\frac{A}{2} s_{0}^{2}\right),
$$

and from eqn (8), for $x_{0}=0$ and $x=L-s_{0}$, one has

$$
s_{0}=L-\int_{s_{0}}^{s\left(L-s_{0}\right)} \frac{d s}{\sqrt{2 C s+\frac{2}{3} B s^{3}+A s^{2}+C_{0}}} .
$$

Then, the axial displacement $s_{L}=s\left(L-s_{0}\right)$ is determinated from eqn (11) and the axial strain $\varepsilon_{L}=\varepsilon\left(L-s_{0}\right)$ follows from eqn $(7)$.

The constitutive parameters $\tau_{0}, a, b$ of the interface frictional model introduced in eqn (1) have been calibrated by fitting the pullout curves provided by the experimental tests performed by Di Maida et al. [17] (Fig. 3) with the theoretical strain-displacement curve obtained from eqns (7), (9) and (11) for the debonding and pullout phases, respectively, and they are listed in Tab. 2. These parameters are found to depend on the embedded length of the fibre. This unexpected behavior is probably due to the progressive abrasion phenomenon occurring at the fibre surface as the pullout process grows. It follows that the pullout behavior depends not only on the nature of the fibre and the matrix but also on the length of the embedded part of the fibre.

It is remarked that in the present Section and in Sec. 2.3, the viscous deformation has not been addressed. However, the experimental tests above mentioned display both the elastic and viscous elongation of the fibre. Therefore, the viscous effects will be considered in Sec. 3 .

\subsection{Applied time-dependent load $F(t)$}

The aforementioned experimental results show the load (in terms of axial strain) vs the displacement during the pullout of the fibre. Such tests have been performed at constant displacement rate $m=1 \mathrm{~mm} / \mathrm{min}$ and thus the time $t$ corresponding to a specific value of the displacement $s_{L}$ can be inferred from the relation $t=s / m$. Based on such a relationship between the external load and time, a piecewise analytical function $F(t)$ of time for the external load can be obtained. In particular, the time-dependent load $F(t)$ has been assumed as a polynomial function that fits adequately the whole averaged load-displacement curve provided by the experimental results reported in [17].

Both the experimental and theoretical curves of the axial strain $\varepsilon_{L}(t)$ vs time $t$ are shown in Fig. 4. Note that the theoretical interpolating piecewise function $\varepsilon_{L}(t)$ consists of two parts. A first one, almost linear and increasing for $t \in\left[0, t_{1}\right]$, reproduces the debonding phase, and a second one, at first increasing and then 


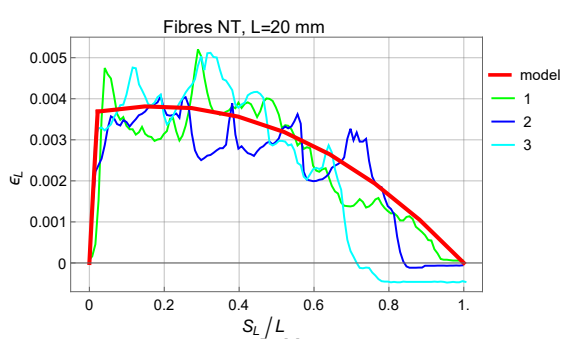

(a) Untreated fibres (NT) with embedded fibre length $L=20 \mathrm{~mm}$.

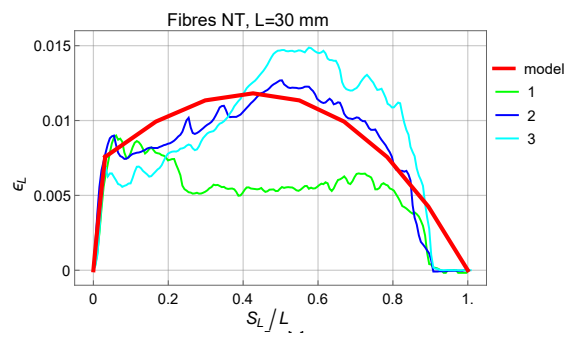

(c) Untreated fibres (NT) with embedded fibre length $L=30 \mathrm{~mm}$.

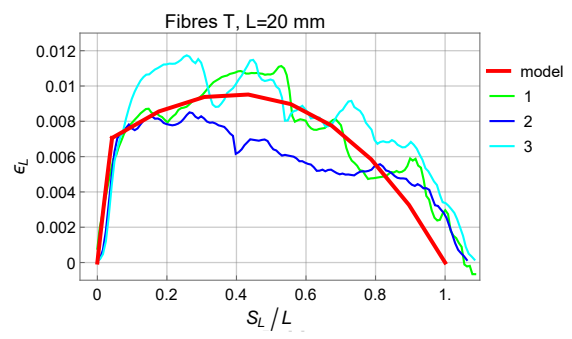

(b) Treated fibres $(\mathrm{T})$ with embedded fibre length $L=20 \mathrm{~mm}$.

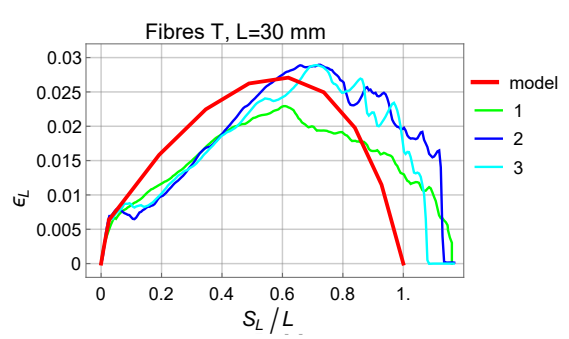

(d) Treated fibres (T) with embedded fibre length $L=30 \mathrm{~mm}$.

Figure 3: Variation of axial strain of the outer part of the fibre $\varepsilon_{L}$ with the normalized displacement $s_{L} / L$ of the actuated fibre cross section: Comparison between the theoretical curve (model) and three experimental curves $(1,2,3)$ for untreated (a), (c) and treated (b), (d) fibres with embedded fibre length $L=20 \mathrm{~mm}$ and $L=30 \mathrm{~mm}$ (from [17]). 
Table 1: Time at the end of the debonding phase $t_{1}$ and at the end of the pullout phase $t_{2}$.

(NT: not treated fibres; T: treated fibres; 20 and $30 \mathrm{~mm}$ : embedded length)

\begin{tabular}{cccccc}
\hline \multicolumn{2}{c}{ Time } & NT $20 \mathrm{~mm}$ & T $20 \mathrm{~mm}$ & NT $30 \mathrm{~mm}$ & T 30 mm \\
\hline$t_{1}$ & {$[\mathrm{~s}]$} & 58.8 & 87.0 & 79.2 & 58.8 \\
$t_{2}$ & {$[\mathrm{~s}]$} & 1046.4 & 1261.8 & 1634.4 & 2097.4 \\
\hline
\end{tabular}

decreasing for $t \in\left[t_{1}, t_{2}\right]$, represents the pullout phase, being $t_{1}$ the time at the end of the debonding and $t_{2}$ the time at the end of the pullout.

The end of the debonding phase and, in turn, the beginning of the pullout process has been assessed numerically from eqn (9) by setting $\lambda=0$, as listed in Tab. 1.

The use of the piecewise analytical function $F(t)$ allows reproducing the whole pullout test accounting for the elastic displacement as well as the viscous counterpart of the fibre, as detailed in Sec. 3. Let

$$
\sigma(t)=\frac{4 F(t)}{\pi d^{2}}
$$

denotes the tensile stress in the outer part of the fibre at time $t$. Then, the axial strain at $x=L-\lambda L$ in the debonding phase follows from eqn (7) as

$$
\varepsilon_{L}(t)=\sqrt{2 C s_{L}(t)+\frac{2}{3} B s_{L}^{3}(t)+A s_{L}^{2}(t)}=\frac{\sigma(t)}{E},
$$

being $F(t)$ the applied load at time $t$. So, for $t \in\left[0, t_{1}\right], s_{L}(t)$ is determined by means of eqn (13), where $\lambda=1$ at time $t=0$ and $\lambda=0$ at $t=t_{1}$. Similarly, for the pullout phase, eqn (7) for $x=L-s_{0}$ can be rewritten in the form

$$
\varepsilon_{L}(t)=\sqrt{2 C s_{L}(t)+\frac{2}{3} B s_{L}^{3}(t)+A s_{L}^{2}(t)+2 C_{0}}=\frac{\sigma(t)}{E},
$$

The introduction of eqn (10) for $C_{0}$ into eqn (14) then provides the following relation between $s_{L}$ and $s_{0}$

$$
2 C\left[s_{L}(t)-s_{0}(t)\right]+\frac{2}{3} B\left[s_{L}(t)-s_{0}(t)\right]^{3}+A\left[s_{L}(t)-s_{0}(t)\right]^{2}=\left[\frac{\sigma(t)}{E}\right]^{2},
$$

that gives $s_{L}(t)$ as a function of $s_{0}(t)$ for $t \in\left[t_{1}, t_{2}\right]$ by solving a cubic equation, being $s_{0}=0$ at time $t=t_{1}$ and $s_{0}=L$ at $t=t_{2}$. Note that the displacement $s_{L}(t)$ takes into account for the rigid body motion $s_{0}(t)$ of the fibre during the pullout stage.

\section{Contribution of viscosity in the outer part of the fibre}

The viscous counterparts of the strain of the fibre during the debonding and the pullout phases are here considered by adopting two different creep functions $[24]$. 


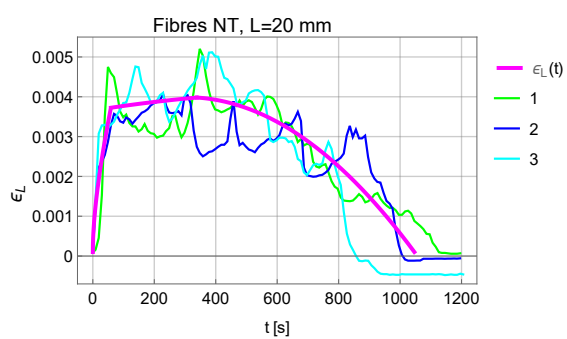

(a) Untreated fibres (NT) with embedded fibre length $L=20 \mathrm{~mm}$.

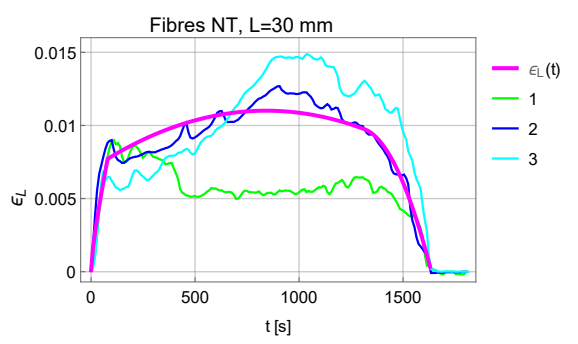

(c) Untreated fibres (NT) with embedded fibre length $L=30 \mathrm{~mm}$.

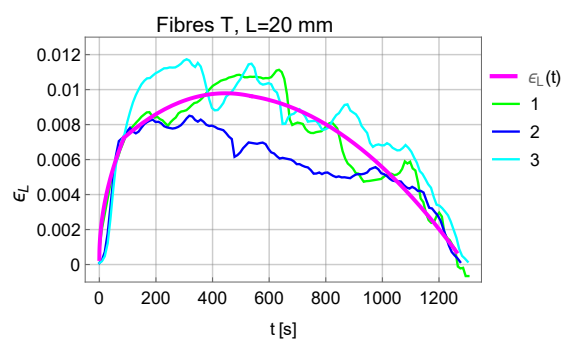

(b) Treated fibres (T) with embedded fibre length $L=20 \mathrm{~mm}$.

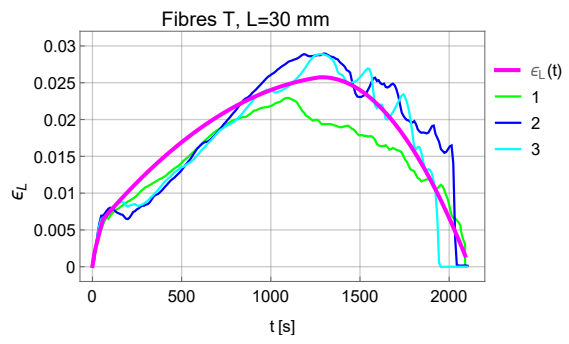

(d) Treated fibres (T) with embedded fibre length $L=30 \mathrm{~mm}$.

Figure 4: Interpolating function of the axial strain $\varepsilon_{L}(t)$ of the outer part of the fibre vs time $t$ and experimental axial strain vs $t$ of three experimental tests $(1$, $2,3)$ for untreated (a), (c) and treated (b), (d) fibres with embedded fibre length $L=20 \mathrm{~mm}$ and $L=30 \mathrm{~mm}$ (from [17]). 
In particular, a creep function based on a fraction-exponential kernel according to the Rabotnov model [25] is considered. Furthermore, in order to compare the results with simplified formulations, an alternative viscoleastic scheme following the SLS model is considered too.

Since the embedded part of the fibre is practically undeformed owing to the constraint offered by the surrounding rigid matrix, then the viscous effects on that part of the fibre have been neglected.

\subsection{Rabotnov model}

A general fraction-exponential function $\psi(t)$ accounting for the viscous deformation of the fibre is considered here. The Rabotnov operator turns out to be

$$
R_{\alpha}(\beta-\tilde{\lambda}, t)=t^{\alpha} \sum_{n=0}^{\infty} \frac{(\beta-\tilde{\lambda})^{n} t^{n(1+\alpha)}}{\Gamma[(n+1)(1+\alpha)]} .
$$

Then, the corresponding creep function reads

$$
\psi(t)=\frac{1}{E_{0}}\left[1-\frac{\tilde{\lambda}}{\beta-\tilde{\lambda}}\left\{M_{1+\alpha}\left[(\beta-\tilde{\lambda}) t^{1+\alpha}\right]-1\right\}\right],
$$

where $M_{a}(z)$ is the well-known Mittag-Leffler function [23, 26]

$$
M_{a}(z)=\sum_{m=0}^{\infty} \frac{z^{m}}{\Gamma(m a+1)},
$$

where $\Gamma(m)=(m-1)$ ! is the Gamma function, $\tilde{\lambda}=\beta\left(E_{0}-E_{\infty}\right) / E_{0}$ and $\alpha$ and $\beta$ are two parameters which have been assessed by fitting properly the results provided by a creep test on PP macrofibres performed by Sorzia [27]. Such parameters are listed in Tab. 3. As known, $\psi_{0}$ denotes the inverse of the istantaneous elastic Young modulus, whereas $\psi_{\infty}$ stands for the inverse of the Young modulus for $t \rightarrow \infty$.

\subsection{SLS model}

A simplified creep function $\psi(t)$ accounting for the viscous deformation of the fibre follows an exponential map according to the classical Zener model

$$
\psi(t)=\psi_{\infty}-\left(\psi_{\infty}-\psi_{0}\right) e^{-\nu t} .
$$

Similarly to the parameters involved in the Rabotnov model, the parameters $\psi_{0}$, $\psi_{\infty}$ and $\nu$ have been set on the basis of creep tests performed on PP macrofibres and reported in Sorzia [27]. The values of such parameters are shown in Tab. 3. The creep function described by eqn (19) corresponds to a Maxwell scheme connected in parallel with an elastic spring, as sketched in Fig. 5(a). It is easy to show that, for this model, the following relationships hold true

$$
\psi_{0}=\frac{1}{k_{1}+k_{2}}, \quad \psi_{\infty}=\frac{1}{k_{2}}, \quad \nu=\frac{k_{1}+k_{2}}{c},
$$




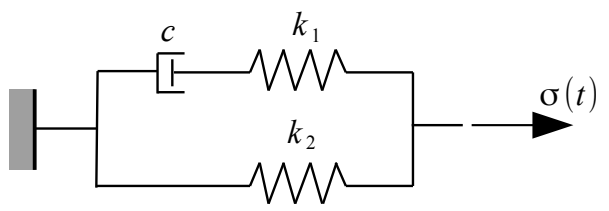

(a) SLS model.
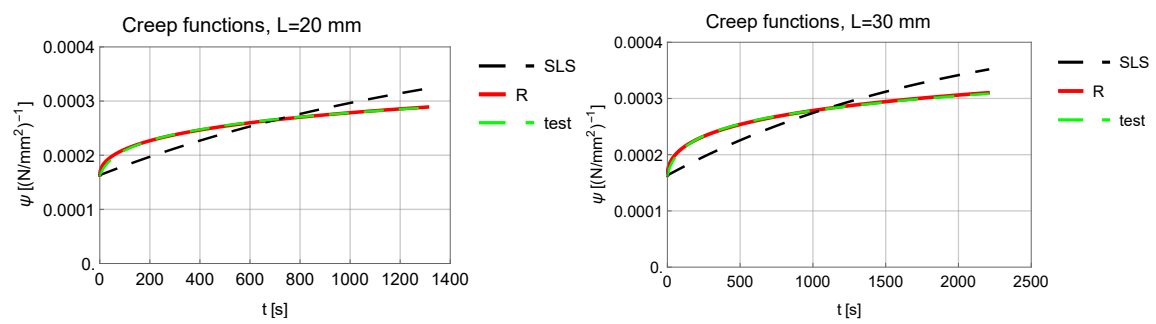

(b) Exponential creep function, Mittag-(c) Exponential creep function, MittagLeffler creep function and creep test until a time over $1200 \mathrm{~s}$. Leffler creep function and creep test until a time over $2000 \mathrm{~s}$.

Figure 5: (a) Sketch of the Standard Linear Solid model (SLS). (b), (c) Comparison between the SLS creep function (SLS), the Rabotnov creep function (R) and the behavior of the PP fibre during the creep test for the time of pullout of embedded fibre length $L=20 \mathrm{~mm}$ and $L=30 \mathrm{~mm}$ (from [27]).

where $k_{1}$ and $k_{2}$ denote the stiffness of the elastic springs in the SLS scheme and $c$ accounts for the dumping effect. Fig. 5(b) displays the comparison between the Rabotnov creep function, the SLS creep function and the experimental creep test in the time range $t \in[0,1200] \mathrm{s}$, whereas Fig. $5(\mathrm{c})$ refers to a time range over 2000 s. Note that the creep function related to the Rabotnov operator fits the experimental results better than the SLS scheme.

\subsection{Viscoelastic strain field of the fibre}

Let $L_{e}$ denote the initial length of the outer part of the fibre between the sample and the actuator and let $z$ be a new abscissa taken from the surface of the sample in the outward direction, namely $z=x-L+s_{0}$, so that $0 \leq z \leq$ $L_{e}+s_{0}$. Based on the superposition principle (i.e. the Boltzmann integral) in the framework of hereditary linear viscoelasticity, the axial strain in the outer part of the fibre turns out to be

$$
\begin{gathered}
\varepsilon_{L}(t)=\sigma(t) \psi_{0}-\int_{t_{z}}^{t} \sigma(\tau) \dot{\psi}(t-\tau) d \tau, \quad \text { for } \quad 0 \leq z \leq s_{L}(t) \\
\varepsilon_{L}(t)=\sigma(t) \psi_{0}-\int_{0}^{t} \sigma(\tau) \dot{\psi}(t-\tau) d \tau, \quad \text { for } \quad s_{L}(t) \leq z \leq s_{L}(t)+L_{e}
\end{gathered}
$$

where $\sigma(t)$ is the tensile stress in the outer part of the fibre at time $t$ defined in eqn (12), $t_{z}$ denotes the time instant when the fibre cross section placed at 
abscissa $z$ at time $t$ came out of the cement matrix at $z=0$ and started to behave viscously, as sketched in Fig. 6(a). Therefore, the viscous deformation at cross section $z$ occurs during the time interval ranging from $t_{z}$ up to the actual time $t$, as computed in the integral in eqn (21). The displacement $u$ of the actuated fibre cross section at time $t$ in the debonding and pullout phases is then given by

$$
u(t)=s_{L}(t)+\int_{0}^{s_{L}(t)} \varepsilon_{L}(t) d z+L_{e} \varepsilon_{L}(t) .
$$

Then, by using the previous results (21) and (22) for $\varepsilon_{L}(t)$ one gets

$$
\begin{aligned}
u(t)=s_{L}(t)+\int_{0}^{s_{L}(t)}\left[\sigma(t) \psi_{0}-\int_{t_{z}}^{t} \sigma(\tau) \dot{\psi}(t-\tau) d \tau\right] d z+ & \\
& +\left[\sigma(t) \psi_{0}-\int_{0}^{t} \sigma(\tau) \dot{\psi}(t-\tau) d \tau\right] L_{e} .
\end{aligned}
$$

In order to evaluate the first integral in eqn (24) for a fixed value of $t$, let us introduce the displacement $s_{L}\left(t_{z}\right)$ of the fibre cross section that was placed at $z=0$ at time $t_{z}$ and is placed at $z$ at time $t$ (see Fig. 6(b)), which is given by the difference

$$
s_{L}\left(t_{z}\right)=s_{L}(t)-z, \quad \text { for } \quad 0 \leq t_{z} \leq t \quad \text { and } \quad 0 \leq z \leq s_{L}(t) .
$$

Then, being the time $t$ fixed, from differentiation of eqn (25) one has

$$
d z=-\dot{s}_{L}\left(t_{z}\right) d t_{z}, \quad \text { for } \quad 0 \leq t_{z} \leq t,
$$

where the over dot denotes the derivative with respect to $t_{z}$. The result (26) allows for the substitution of $d z$ into eqn (24), by considering that $t_{z}=0$ for $z=s_{L}(t)$ and $t_{z}=t$ for $z=0$, thus giving

$$
\begin{aligned}
u(t)=s_{L}(t)+\int_{0}^{t}\left[\sigma(t) \psi_{0}-\int_{t_{z}}^{t} \sigma(\tau) \dot{\psi}(t-\tau) d \tau\right] \dot{s}_{L}\left(t_{z}\right) d t_{z}+ \\
+\left[\sigma(t) \psi_{0}-\int_{0}^{t} \sigma(\tau) \dot{\psi}(t-\tau) d \tau\right] L_{e},
\end{aligned}
$$

where the first integral can be evaluated as shown in Appendix.

Note that $s_{L}\left(t_{z}\right)$ is known once the hystory of $s_{L}(t)$ has been evaluated up to time $t \geq t_{z}$, whereas the time $t_{z}$ can be evaluated as a function of $t$ by inverting the map (25), see Fig. 6(b). The displacement $s_{L}(t)$ is evaluated through the model reported in Sec. 2.2 based on the eqns (7), (9) and (11) in debonding and pullout phase, respectively, using the applied load $F(t)$ as found in Sec. 2.3.

The model of Sec. 2.2 accounts for the elastic contribution of the displacement $s_{L}$ in debonding and pullout phase, but it does not account for the elastic contribution of the rigid motion $s_{0}$ at the pullout stage, which has been added apart. Eqn (27) allows evaluating straightforwardly the displacement of the end of the fibre based on the map $\dot{s_{L}}\left(t_{z}\right)$. 


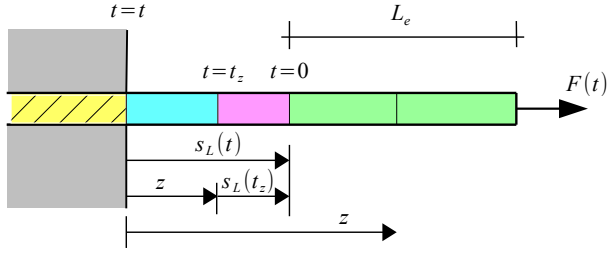

(a)

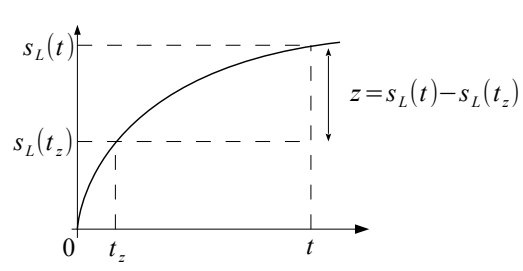

(b)

Figure 6: (a) Sketch of the elongation of the outer part of the fibre due to viscoelastic effects.

(b) Qualitative variation of displacement $s_{L}\left(t_{z}\right)$ with time $t_{z}$ for $z=s_{L}(t)-$ $s_{L}\left(t_{z}\right)$.

Table 2: Parameters of the constitutive law involved in eqn (1).

(NT: not treated fibres; T: treated fibres; 20 and $30 \mathrm{~mm}$ : embedded length)

\begin{tabular}{|c|c|c|c|c|}
\hline Parameter & NT $20 \mathrm{~mm}$ & T $20 \mathrm{~mm}$ & $\mathrm{NT} 30 \mathrm{~mm}$ & T $30 \mathrm{~mm}$ \\
\hline$\tau_{0} \quad\left[\mathrm{~N} / \mathrm{mm}^{2}\right]$ & 0.22 & 0.42 & 0.3 & 0.25 \\
\hline$a \quad\left[\mathrm{~N} / \mathrm{mm}^{3}\right]$ & 0.016 & 0.065 & 0.04 & 0.122 \\
\hline$b \quad\left[\mathrm{~N} / \mathrm{mm}^{4}\right]$ & $1.0 \times 10^{-5}$ & $1.0 \times 10^{-5}$ & $1.0 \times 10^{-5}$ & 0.003 \\
\hline
\end{tabular}

Table 3: Property of PP fibre and relevant data concerning the pullout test.

\begin{tabular}{|c|c|}
\hline Property & Value \\
\hline Diameter $d \quad[\mathrm{~mm}]$ & 0.78 \\
\hline Tensile strength $\left[\mathrm{N} / \mathrm{mm}^{2}\right]$ & 273 \\
\hline Elastic Young modulus $E \quad\left[\mathrm{~N} / \mathrm{mm}^{2}\right]$ & $4.591 \times 10^{3}$ \\
\hline Elastic Young modulus $E_{0}$ at $t=0 \quad\left[\mathrm{~N} / \mathrm{mm}^{2}\right]$ & $4.591 \times 10^{3}$ \\
\hline Elastic Young modulus $E_{\infty}$ at $t \rightarrow \infty \quad\left[\mathrm{N} / \mathrm{mm}^{2}\right]$ & $2.246 \times 10^{3}$ \\
\hline Creep modulus $\psi_{0}$ at $t=0 \quad\left[\left(\mathrm{~N} / \mathrm{mm}^{2}\right)^{-1}\right]$ & $1 /\left(4.591 \times 10^{3}\right)$ \\
\hline Creep modulus $\psi_{\infty}$ at $t \rightarrow \infty \quad\left[\left(\mathrm{N} / \mathrm{mm}^{2}\right)^{-1}\right]$ & $1 /\left(2.246 \times 10^{3}\right)$ \\
\hline$\alpha \quad[-]$ & $-1 / 3$ \\
\hline$\beta \quad\left[\mathrm{s}^{-(1+\alpha)}\right]$ & -0.00608 \\
\hline$\nu \quad\left[\mathrm{s}^{-1}\right]$ & 0.00037 \\
\hline Rate of the actuator in pullout tests $m$ & 1 \\
\hline Embedded fibre length $L \quad[\mathrm{~mm}]$ & 20 and 30 \\
\hline Outer fibre length $L_{e} \quad[\mathrm{~mm}]$ & 110 \\
\hline
\end{tabular}




\section{Results}

The strain vs displacement curves provided by the proposed theoretical model have been compared with the experimental curves provided by Di Maida et al. [17]. Such tests were carried out both on plain fibres and fibres treated with nano-silica embedded in a cementitious matrix for a total length $L=20 \mathrm{~mm}$ and $L=30 \mathrm{~mm}$. The length of the outer part of the fibres was $L_{e}=110 \mathrm{~mm}$, which coincides with the distance between the surface of the cement sample and the actuator grip. The geometric and mechanical parameters of the PP fibres and some relevant setup details are listed in Tab. 3.

The curves plotted in Fig. 7 show that the theoretical predictions closely fit the experimental results provided by the pullout test performed both on untreated and treated PP macrofibres. It is worth noting that both creep functions considered in the present investigation allows reproducing closely the experimental results in terms of axial strain $\varepsilon_{L}(t)$ of the outer part of the fibre vs its axial displacement $u / L$. Note also that the experimental curves shown in Fig. 7(a),(c) exhibit a small plateau with vanishing pullout resistance before the complete pullout. This is probably due to the fact that the load cell cannot measure accurately the sudden loss of the external load occurring for the untreated fibres near the end of the test.

The curves reported in Fig. 7(b),(d) for treated fibres show that the external load driving the pullout phase exhibits a remarkable increasing with respect to untreated fibres, owing to the improvement in the bond strength. This is due to abrasion phenomena occurring on the fibre surface during the pullout test. Indeed, as shown in Figs. 7, the peak value of the treated fibres is more than twice than that recorded for untreated ones. It is worth noting that the predicted response of the untreated fibres exhibits a plateau just after the debonding phase. Conversely, the theoretical prediction of the treated fibres exhibits a peak value in correspondance of the dimensionless axial displacement $u / L \simeq 0.4$ for $L=20 \mathrm{~mm}$ and $u / L \simeq 0.8$ for $L=30 \mathrm{~mm}$.

Furthermore, the computed axial displacement $u(t)$ has been compared with the displacement recorded during the experimental tests. This comparison is shown in Fig. 8. Note that the constant displacement rate of the actuator during the test was $m=1 \mathrm{~mm} / \mathrm{min}$, so that the imposed displacement at the end section of the fibre during time was $m \cdot t$ and the tests continued for $1200 \mathrm{~s}$ and $1800 \mathrm{~s}$, being $L=20 \mathrm{~mm}$ and $L=30 \mathrm{~mm}$ the embedded fibre length respectively.

As shown in Figs. 8(a),(c) for untreated fibres, there is a gap between the theoretical and experimental curves that increases with time until a time of about $1046 \mathrm{~s}$ and $1634 \mathrm{~s}$ for $L=20 \mathrm{~mm}$ and $L=30 \mathrm{~mm}$ of embedded fibre length respectively, namely at a time shorter than the effective duration of the test. This difference is due to the fact that the interpolating function of the load $F(t)$ drops at that time (see Figs. 4(a),(c)), when the fibre does not exhibit any more pullout strength, even though the complete extraction of the fibre has not been achieved. This justifies the fact that, at the time of $1046 \mathrm{~s}$ and $1634 \mathrm{~s}$, the theoretical model predicts a displacement longer than $20 \mathrm{~mm}$ and $30 \mathrm{~mm}$ whereas, at the same time, the actuator reached a displacement of about 


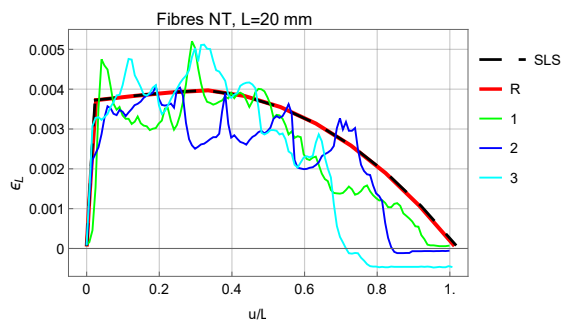

(a) Untreated fibres (NT) with embedded fibre length $L=20 \mathrm{~mm}$.

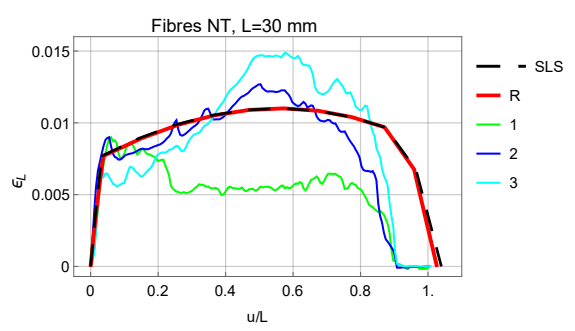

(c) Untreated fibres (NT) with embedded fibre length $L=30 \mathrm{~mm}$.

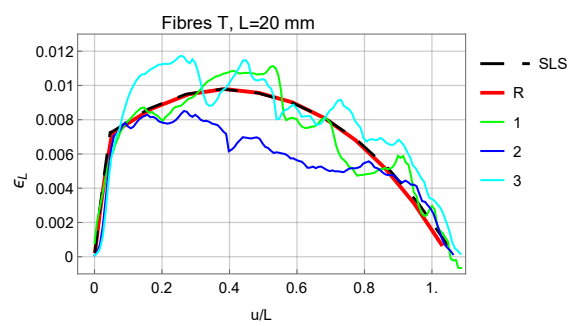

(b) Treated fibres $(\mathrm{T})$ with embedded fibre length $L=20 \mathrm{~mm}$.

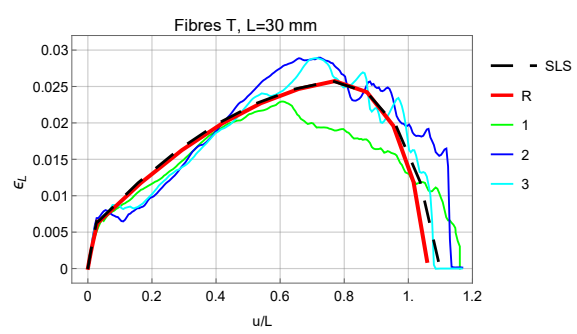

(d) Treated fibres (T) with embedded fibre length $L=30 \mathrm{~mm}$.

Figure 7: Variation of axial strain $\varepsilon_{L}$ of the outer part of the fibre vs $u / L$ : Comparison between the theoretical model (SLS with SLS creep function, R with Rabotnov creep function) and three experimental curves $(1,2,3)$ for untreated (a), (c) and treated (b), (d) fibres with embedded fibre length $L=20 \mathrm{~mm}$ and $L=30 \mathrm{~mm}$ (from [17]).

$17.5 \mathrm{~mm}$ and $27.5 \mathrm{~mm}$ respectively.

Concerning the treated fibres with $L=20 \mathrm{~mm}$ (Fig. 8(b)) embedded fibre length, there is a very small gap between the theoretical and experimental curves just at the beginning of the test, namely at the debonding phase. This can be ascribed to the fact that the recorded data are not sufficiently accurated during the initial stage of the test, in which the fibre detaches from the matrix. Conversely, as shown in Fig. 8(d) for treated fibres with $L=30 \mathrm{~mm}$, there is a certain gap between predictions and experimental results near the end of the test.

\section{Concluding remarks}

An analytical formulation of the pullout problem of a viscoelastic fibre embedded in a cementitiuous matrix has been proposed in the present work. Based on proper creep functions, the proposed model allows evaluating the whole pullout process of synthetic macrofibres accounting for the viscoelastic strain of the outer part of the fibre. 


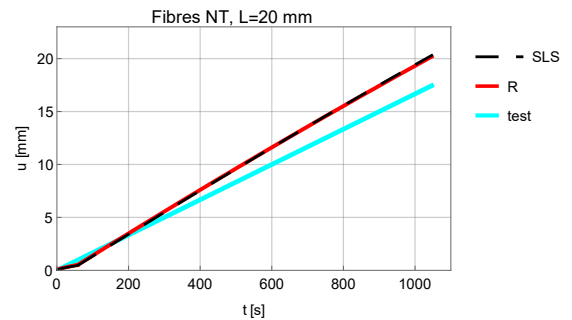

(a) Untreated fibres (NT) with embedded fibre length $L=20 \mathrm{~mm}$.

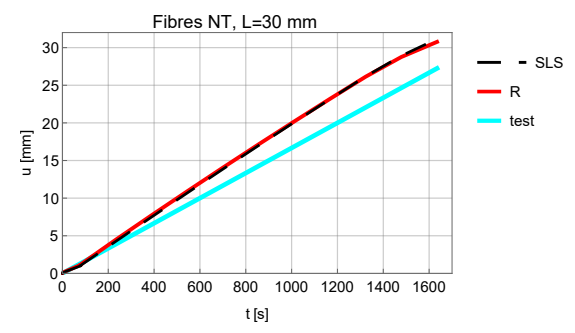

(c) Untreated fibres (NT) with embedded fibre length $L=30 \mathrm{~mm}$.

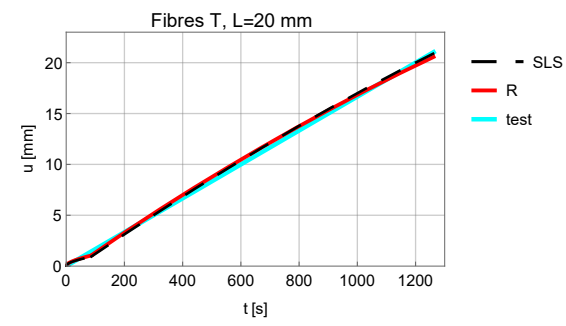

(b) Treated fibres (T) with embedded fibre length $L=20 \mathrm{~mm}$.

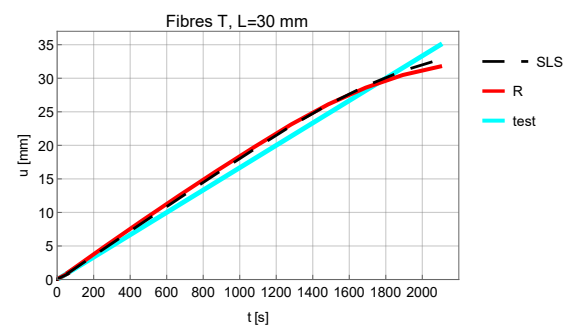

(d) Treated fibres ( $\mathrm{T})$ with embedded fibre length $L=30 \mathrm{~mm}$.

Figure 8: Displacement $u(t)$ of the actuated fibre cross section during time: Comparison between the proposed model (SLS with SLS creep function, $\mathrm{R}$ with Rabotnov creep function) and experimental tests (1, 2, 3) for untreated (a), (c) and treated (b), (d) fibres with embedded fibre length $L=20 \mathrm{~mm}$ and $L=30 \mathrm{~mm}$ (from [17]). 
Three parameters $\tau_{0}, a, b$ fully characterize the interfacial frictional stress as a monotonic function of the slippage, which reveals adequate for properly describing the adhesion properties of both treated and untreated fibres. The same model can be used for modelling the pullout behavior of other kinds of synthetic fibres as well as the softening pullout response of steel fibres, provided that the constitutive parameters of the interface are selected properly. It is remarked that the model is able to evaluate not only the elastic elongation, but also the viscous effects of the fibre during the debonding and pullout phases.

The proposed analytical model can be used as a valid tool for predicting the post-cracking behavior of FRC structures. In particular, by assuming a uniform distribution of the fibre on the cross section of a sample, accordingly, by defining a proper moment-curvature relationship starting from the proposed pullout model, the equivalent flexural strength and, in turn, the mechanical behavior of FRC structures near their limit state can be properly assessed, with particular reference to bent beams [28, 29] and Kirchhoff plates [30, 31, 32, 33, 34]. Indeed, an experimental evidence of the benefits induced by the fibre treatrement with nanosilica on the post cracking residual strength of FRC elements has been investigated by Di Maida et al.[18] through three-point loading bending tests on beam-like samples. As a further example, the proposed approach could be used to predict time-dependent crack mouth opening displacement of FRC beam-like notched samples subjected to three-point loading bending tests and to simulate the creep behavior of bent beams in post-cracking state [35]. In these cases indeed, the present model can capture the delayed deformation due to the viscous relaxation of the fibres bridging the crack surfaces.

The evaluation of the viscous strain in the embedded part of the fibre during its pullout represents a complex challenge as it involves moving boundary conditions, that the Authors wish to address in a forthcoming work.

\section{Appendix}

The first integral in eqn (27) can be evaluated by considering finite time instants. Let $I(t)$ denote the first integral in eqn (27), namely

$$
I(t)=\int_{0}^{t}\left[\sigma(t) \psi_{0}-\int_{t_{z}}^{t} \sigma(\tau) \dot{\psi}(t-\tau) d \tau\right] \dot{s}_{L}\left(t_{z}\right) d t_{z} .
$$

Integrating by parts the integral in $d \tau$ in eqn (28) one obtains

$$
I(t)=\int_{0}^{t}\left[\sigma\left(t_{z}\right) \psi\left(t-t_{z}\right)+\int_{t_{z}}^{t} \dot{\sigma}(\tau) \psi(t-\tau) d \tau\right] \dot{s}_{L}\left(t_{z}\right) d t_{z},
$$

namely

$$
I(t)=\int_{0}^{t}\left[\int_{0}^{t_{z}} \dot{\sigma}(\tau) \psi\left(t-t_{z}\right) d \tau+\int_{t_{z}}^{t} \dot{\sigma}(\tau) \psi(t-\tau) d \tau\right] \dot{s}_{L}\left(t_{z}\right) d t_{z},
$$


or equivalently

$$
I(t)=\int_{s(0)}^{s_{L}(t)}\left[\int_{\sigma(0)}^{\sigma\left(t_{z}\right)} d \sigma(\tau) \psi\left(t-t_{z}\right)+\int_{\sigma\left(t_{z}\right)}^{\sigma(t)} d \sigma(\tau) \psi(t-\tau)\right] d s_{L}\left(t_{z}\right) .
$$

By considering a finite number of time instants $t_{i}$ for $i=0,1, \ldots, n$, with $t_{0}=0$ and $t_{n}=t$, and the corresponding increments of the axial displacement $\Delta s_{i}=s_{L}\left(t_{i}\right)-s_{L}\left(t_{i-1}\right)$ and tensile stress $\Delta \sigma_{i}=\sigma\left(t_{i}\right)-\sigma\left(t_{i-1}\right)$, then the integral $I(t)$ at time $t$ can be evaluated by the following sum

$$
I(t)=\sum_{j=1}^{n} \Delta s_{j}\left[\sum_{i=1}^{j} \Delta \sigma_{i} \psi\left(t-t_{j}\right)+\sum_{i=j+1}^{n} \Delta \sigma_{i} \psi\left(t-t_{i}\right)\right], \quad \text { for } \quad t_{n} \leq t .
$$

\section{Acknowledgements}

Support from "Gruppo Nazionale di Fisica Matematica - INdAM" is gratefully acknowledged.

\section{COI statement}

The authors declare that the present work has been realized in compliance with the Ethical Standards.

This study was funded by the aforementioned grant only.

Conflict of Interest: The authors declare that they have no conflict of interest.

[1] D. Scerrato, I. Giorgio, A. Madeo, A. Limam, F. Darve, A simple non-linear model for internal friction in modified concrete, International Journal of Engineering Science 80 (2014) 136-152.

[2] L. Dezi, G. Menditto, A. M. Tarantino, Homogeneous structures subjected to successive structural system changes, ASCE Journal of Engineering Mechanics 116(8) (1990) 1723-1732.

[3] L. Dezi, G. Menditto, A. M. Tarantino, Viscoelastic heterogeneous structures with variable structural system, ASCE Journal of Engineering Mechanics 119(2) (1993) 238-250.

[4] L. Lanzoni, A. Nobili, A. M. Tarantino, Performance evaluation of a polypropylene-based draw-wired fibre for concrete structures, Construction and Building Materials 28 (2012) 798-806.

[5] A. Bentur, S. Mindess, Fibre reinforced cementitious composites, Taylor \& Francis. 
[6] A. Brandt, Fibre reinforced cement-based (frc) composites after over 40 years of development in building and civil engineering, Composite Structures 86 (2008) 3-9.

[7] A. Nobili, L. Lanzoni, A. M. Tarantino, Experimental investigation and monitoring of a polypropylene-based fiber reinforced concrete road pavement, Construction and Building Materials 47 (2013) 888-895.

[8] A. Nobili, F. O. Falope, Impregnated carbon fabric-reinforced cementitious matrix composite for rehabilitation of the finale emilia hospital roofs: case study, Journal of Composites for Construction 21 (4) (2017) 05017001.

[9] C. Signorini, A. Nobili, F. O. Falope, Mechanical performance and crack pattern analysis of aged carbon fabric cementitious matrix (cfrcm) composites, Composite Structures 202 (2018) 1114-1120.

[10] F. O. Falope, L. Lanzoni, A. M. Tarantino, Modified hinged beam test on steel fabric reinforced cementitious matrix ( $\mathrm{sfrcm}$ ), Composites Part B: Engineering 146 (2018) 232-243.

[11] F. O. Falope, L. Lanzoni, A. M. Tarantino, Double lap shear test on steel fabric reinforced cementitious matrix (sfrcm), Composite Structures 201 (2018) 503-513.

[12] L. R. Betterman, C. Ouyang, S. Shah, Fibre-matrix interaction in microfibre reinforced mortar, Advanced Cement-Based Materials 2 (1995) 53-61.

[13] N. Banthia, N. Nandakumar, Crack growth resistance of hybrid fibre composites, Journal of Cement Composites 25(1) (2003) 3-9.

[14] J. A. O. Barros, J. A. Figueiras, Flexural behavior of sfrc: testing and modelling, Journal of Materials in Civil Engineering 11(4) (1999) 331-339.

[15] J. A. O. Barros, Post-cracking behavior of steel fiber reinforced concrete, Materials and Structures 38 (2005) 47-56.

[16] V. M. C. F. Cunha, J. A. O. Barros, J. M. Sena-Cruz, Pullout behaviour of hooked-end steel fibres in self-compacting concrete, Report 07-DEC/E06.

[17] P. Di Maida, E. Radi, C. Sciancalepore, F. Bondioli, Pullout behavior of polypropylene macro-synthetic fibers treated with nano-silica, Construction and Building Materials 10 (82) (2015) 39-44.

[18] P. Di Maida, C. Sciancalepore, E. Radi, F. Bondioli, Effects of nano-silica treatment on the flexural post cracking behavior of polypropylene macrosynthetic fibre reinforced concrete., Mechanics Research Communications 88 (2018) 12-18.

[19] A. E. Naaman, G. G. Namur, A. J. M., N. H. S., Fiber pullout and bond slip. i: Analytical study, J. Struct. Eng. 117 (1991) 2769-2790. 
[20] V. M. C. F. Cunha, J. A. O. Barros, J. M. Sena-Cruz, Pullout behavior of steel fibers in self-compacting concrete, Journal of Materials in Civil Engineering 22 (1) (2010) 1-9.

[21] W. C. Choi, S. J. Jang, H. D. Yun, Interface bond characterization between fiber and cementitious matrix, International Journal of Polymer Science Article ID 616949 (2015) 11p.

[22] E. Radi, L. Lanzoni, A. Sorzia, Analytical modelling of the pullout behavior of synthetic fibres treated with nano-silica, Procedia Engineering 109 (2015) $525-532$.

[23] I. Sevostianov, V. Levin, E. Radi, Effective viscoelastic properties of shortfiber reinforced composites, International Journal of Engineering Science 100 (2016) 61-73.

[24] R. M. Christensen, Theory of Viscoelasticity. An Introduction., Academic Press, 1982, 2nd ed.

[25] Y. N. Rabotnov, Elements of Hereditary Solid Mechanics, Mir, 1977.

[26] R. Gorenflo, A. Kilbas, F. Mainardi, S. Rogosin, Mittag-Leffler Functions, Related Topics and Applications, Springer-Verlag, 2014.

[27] A. Sorzia, Modelling of creep and stress relaxation test of a polypropylene micro-fibre by using fraction-exponential kernel, Modelling and Simulation in Engineering 2016 (2016) 1-7.

[28] M. N. Soutsos, T. T. Le, A. P. Lampropoulos, Flexural performance of fibre reinforced concrete made with steel and synthetic fibres, Construction and Building Materials 36 (2012) 704-710.

[29] A. Meda, F. Minelli, G. A. Plizzari, Flexural behaviour of rc beams in fibre reinforced concrete, Composites Part B: Engineering 43(8) (2012) 29302937.

[30] L. Lanzoni, A. Nobili, E. Radi, A. Sorzia, Axisymmetric loading of an elasticplastic plate on a general two-parameter foundation, J. Mech. Mat. Struct. 10 (2015) 459-479.

[31] E. Radi, P. Di Maida, Analytical solution for ductile and frc plates on elastic ground loaded on a small circular area, J. Mech. Mat. Struct. 9 (2014) 313-331.

[32] A. Nobili, E. Radi, L. Lanzoni, A cracked infinite kirchhoff plate supported by a two-parameter elastic foundation, J. Eur. Ceramic Soc. 34 (2014) $2737-2744$.

[33] L. Lanzoni, A. Nobili, E. Radi, A. Sorzia, Failure mechanism of frc slabs on non-local ground, Meccanica 10 (2016) 1-20. 
34] L. Lanzoni, E. Radi, A. Nobili, Ultimate carrying capacity of elastic-plastic plates on a pasternak foundation, Journal of Applied Mechanics 81 (2014) $1-9$.

[35] A. J. Babafemi, Tensile creep of cracked macro synthetic fibre reinforced concrete, 2015, phD dissertation. 